\title{
Digital Wallet
}

\author{
Harshal R. Kanhekar ${ }^{\mathrm{a}}$, Mrs. Sayali N. Mane ${ }^{\mathrm{a}}$ \\ ${ }^{a}$ Department of Communication Network, Pune University, Pune-411044, India
}

\begin{abstract}
As the world is getting digital there are few things which are used as it is for a long time. The most important thing which we do in our daily life is the transaction of money. Money transaction is a process which should be digitized for more security and the ease of the individual. So, by making a digital wallet the money transaction can be made much simpler and effortless. Using digital wallet we can keep all our important cards such as health card, driving license and other important papers in only one place with much more security.

The digital wallet can be our transaction medium which can be used anywhere such as shops, malls or for giving money to someone else. And with such high security such as finger print scanning our money and all the information which the wallet is carrying will be safe. In case, if the wallet is lost GPS will help to detect the location of our device and that what makes the wallet a digital safe.
\end{abstract}

Index Terms: Secure Money Transaction, e-Money, RFID, AES.

(C) 2015 Published by MECS Publisher. Selection and/or peer review under responsibility of the Research Association of Modern Education and Computer Science

\section{Introduction}

A digital wallet refers to an electronic device that allows an individual to make electronic commerce transactions. This can include purchasing items at a store. Increasingly, digital wallets are being made not just for basic financial transactions but to also authenticate the holder's credentials. For example, a digital-wallet could potentially verify the age of the buyer to the store while purchasing alcohol. It is useful to approach the term "digital wallet" not as a singular technology but as three major parts: the system (the electronic infrastructure) and the application (the software that operates on top) and the device (the individual portion). [1]

An individual's bank account can be linked to the digital wallet. They might also have their driver's license, health card, loyalty card(s) and other ID documents stored on the PC. RFID Tag is used for the identity. The credentials can be passed to a merchant's terminal wirelessly via RF Module. [2]

A digital wallet has both a software and information component. The software provides security and encryption for the personal information and for the actual transaction. Typically, digital wallets are stored on

* Corresponding author.

E-mail address: harshalkanhekar@gmail.com, sayalimane@rediffmail.com 
the client side and are easily self-maintained and fully compatible. Digital wallets are gaining popularity among major retailers due to the security, efficiency and added utility it provides to the end-user, which increases their satisfaction of their overall purchase. The information component is basically a database of user-inputted information. The key point to take from digital wallets is it composed of both digital wallet devices and digital wallet systems.

The Digital Wallet will be a hardware device which will be using Radio Frequency (RF) for the exchange of money. Using this device we can make payments at the store without carrying any hard cash with us. The other components used in the digital wallet will be ARM7 processer, matrix display and keyboard. There will a finger print scanner for the purpose of security. GPS will also be used in the device as a safety feature for the wallet which will help to locate the device and security features. [4]

For the security purpose and for avoiding the clone attackers to hack into the device the Advance Encryption Standard (AES) has been used for encryption purpose. AES is a block cipher with a block length of 128 bits. It allows for different key lengths such as 128, 192 and 256 bits. Encryption consists of 10 rounds of processing for 128-bit keys, 12 rounds for 192-bit keys and 14 rounds for 256-bit keys. Through AES, the data can be transmitted and received in a very secure way. AES keeps the code encrypted so that, it cannot be hacked by any clone attacker. [3]

The basic function of the digital wallet will be for the transaction in the store or to other people, but there are several other uses of the digital wallet. The wallet can be used as a key for the vehicles. There will be a soft copy of the license in the wallet which will be acting as a pass. It will also help to keep our license in check. In the wallet other information such as our health information can be stored which can be stored which will be helpful in the cases of the emergency. [5]

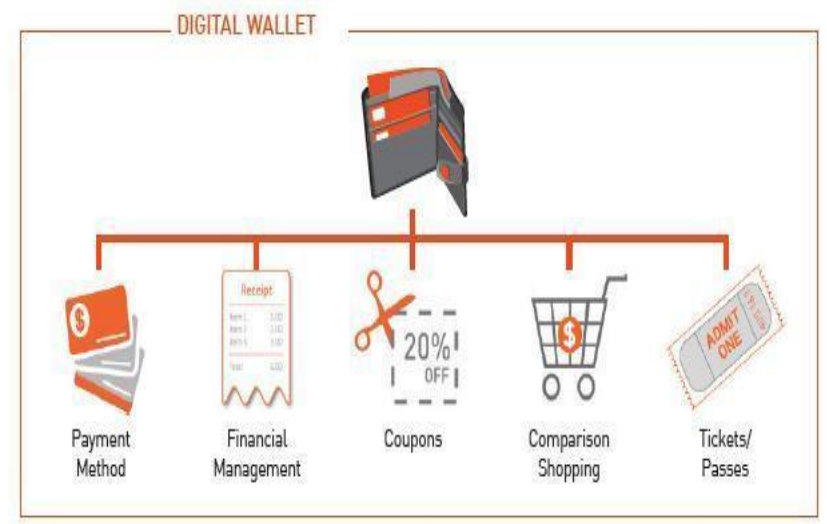

Fig.1. Digital Wallet and its Uses

\section{Digital Wallet Ecosystem}

An understanding of key stackholders involved in the efforts to make mobile payment, loyalty and digital wallets universal realities leads to a better appreciation of the complexity of the challenge.

First there are those who own the card or account date. There are many type of card controller, including the various type of incumbent payment vehicle networks such as Visa, MasterCard, Discover, American Express, etc. that are working hard to remain relevant as acceptance of mobile payments grows among merchants and consumers. These networks set the rule for accounts and license issuers to distribute the account to the general population so more transaction run over the network. The quality of a mobile payments application is critical to successful consumer take-up. However, banks can no longer rely on manually testing mobile payments applications as there are too many variables. For mobile payments, test automation is almost always necessary. 
It's too complicated to mitigate a significant number of risks, diverse hardware, software and network combinations as well as the many test data permutations to be exercised. An effective test automation strategy with clear requirements, rationalization of devices and limiting the number of combinations of operating system will provide clear benefits such as accelerating speed to market, significant savings and proven practices that embrace consumer expectations.

In the fast changing market, companies are challenged to apply an approach that will provide sufficient test coverage of devices, operating systems and network operators enabling them to launch m-payments quickly and within budget. The most effective approaches are testing earlier in the development cycle and using both test tools, agile methodology, open source approach and automation frameworks. Spending time on test management will help you to create a multi-channel strategy embracing quality assurance, risk based plan and a systematic approach to improve efficiency. Pragmatic test management also plays a key part in adopting dynamic and agile strategy with embed Open Source approach, helping organisations increase efficiency and to accelerate speed to market.

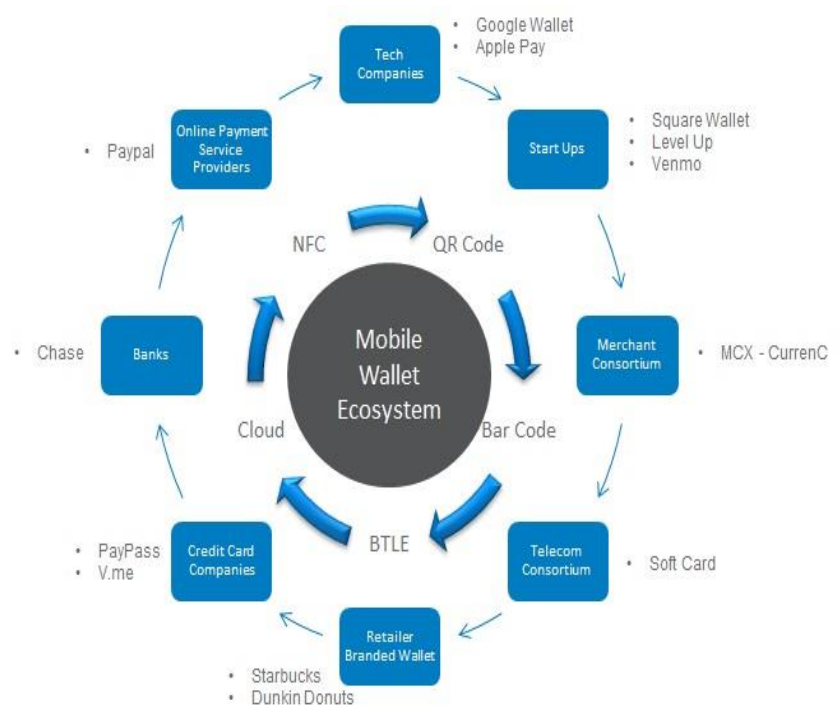

Fig.2. Digital Wallet Ecosystem

\section{System Design}

The Digital Wallet uses RF for the transaction and billing. As RF is well known wireless technology and short distance communication can be done very easily using this technology, RF is used for transaction purposes. The hardware comprises of three parts:

- Digital Wallet(Unit 1)

- Billing Unit(Unit 2)

- Bank Server(Unit 3)

These three units together form a complete scenario of billing using the digital wallet. Firstly, the digital wallet is the mobile device which is used for the payment in the shopping mall. This digital wallet consists of ARM7 controller, LCD, Matrix Keyboard, Fingerprint scanner. The function of this unit is for the authentication of the user and for payment purposes. The wallet consists of fingerprint scanner which is used 
for authentication. Every time when a transaction is to done, the wallet asks for the user authentication from the fingerprint scanner. If the user is registered, the wallet will automatically do the transaction.

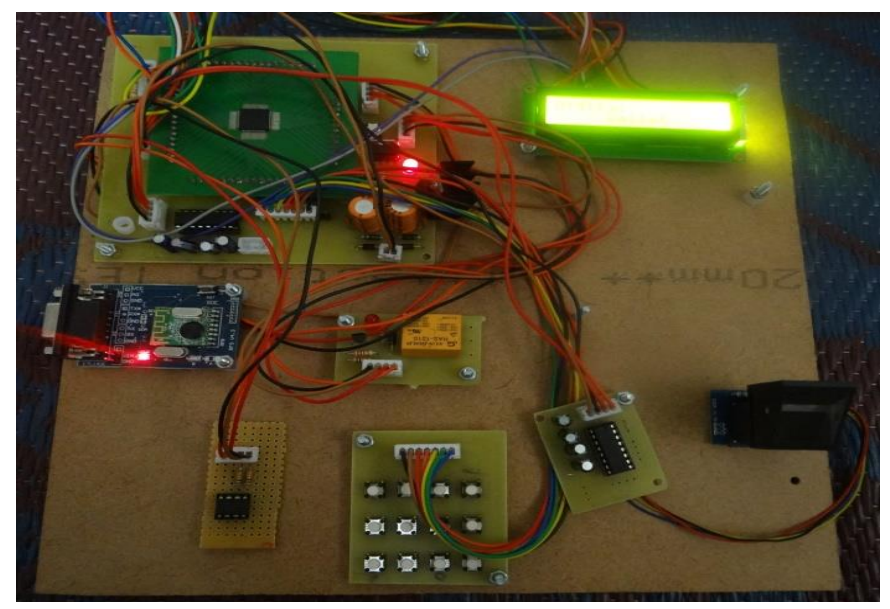

Fig.3. Digital Wallet with Fingerprint Scanner

In this particular shopping process RFID tags are used as items for the shopping purposes. The billing unit is considered as the shopping mall were different items can be purchased. This unit consists of ARM7 controller, RFID reader. It also needs a Visual Basic interface for showing the billing section. As soon as the RFID tags are placed on the RFID reader, the items are identified and the items full detail is shows on the billing window. The billing section then generates the total amount which has to be paid and the list of the items purchased. The digital wallet is then used for the payment process. Only the users authentication is required using the fingerprint scanner. As soon as the fingerprint scanner detects the user, the payment is done. After the payment a message is received that the amount has been transferred to the billing section.

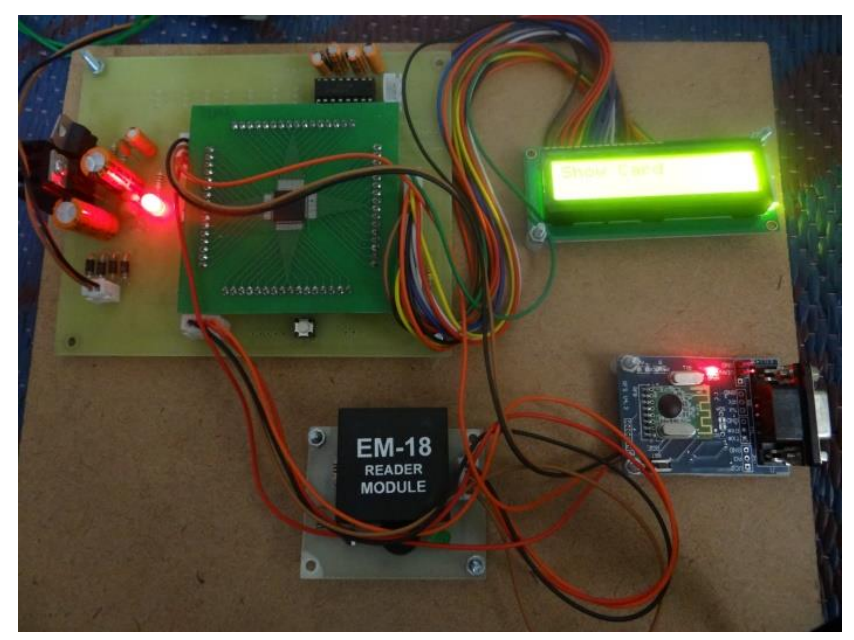

Fig.4. Billing Unit with RFID reader

The third unit is the Bank server. It consists of RFID and GSM. This unit acts as a bank account of the user and whenever user needs to recharge the wallet, user can transfer the money to the digital wallet. The Bank 
server is shown using VB interface were the user can enter the amount to be recharged in the wallet. When the amount is being transferred, the transmission is encrypted using AES algorithm. 128-bit key is used for the authentication of the transferred amount. Thus the user account and the received amount is safe from the hackers. When the amount is received the user will receive a text message on mobile device that particular amount has been deducted from the users account. Thus the user will know that the amount has been successfully transferred to the wallet.

\section{Implementation}

Fig.3 shows the prototype and hardware diagram of the proposed system. The main processor part uses ARM7 controller. This part is very important as it controls all the function of the digital wallet as well as the billing unit for the shopping mall. In order to show the feasibility of the proposed system, VB (Visual Basic) is used to show the different interface of the shopping unit as well as the bank server unit. VB provides minimalistic API which is easy to use and modification can be done very easily in the interface.

The VB interface for the Billing unit for mall is made to show the shopping scenario in the mall. The interface consists of the wallet number, billing details, quantity and the total amount. The items to be purchased can be edited in this interface. New items can be added and named and priced in this interface. While shopping the user has to place the items tag near the RFID scanner and the item is detected and is shown in the billing details section. The quantity of the items is also shown. After the shopping, total amount is shown next to the billing details. The user can make the payment as soon as the get bill button is clicked. Thus the payment is made from the user side which is easier and convenient.

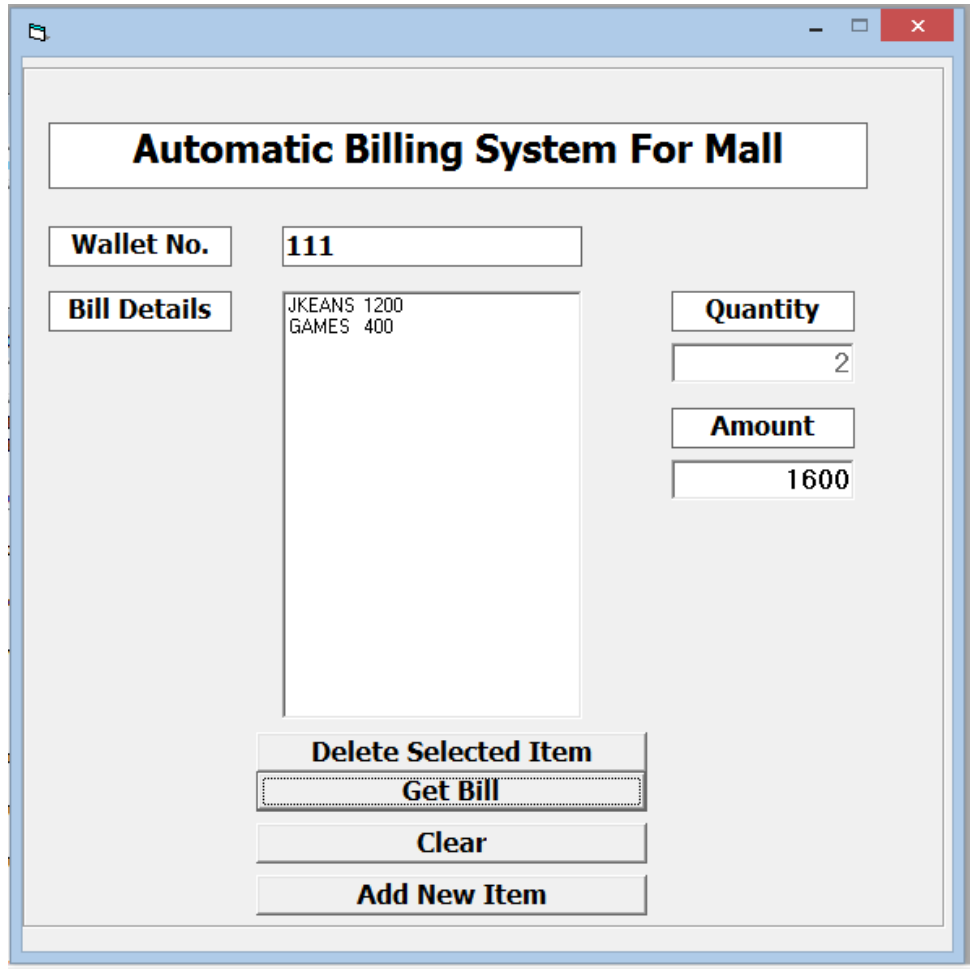

Fig.5. VB interface for the Billing Unit for Mall 


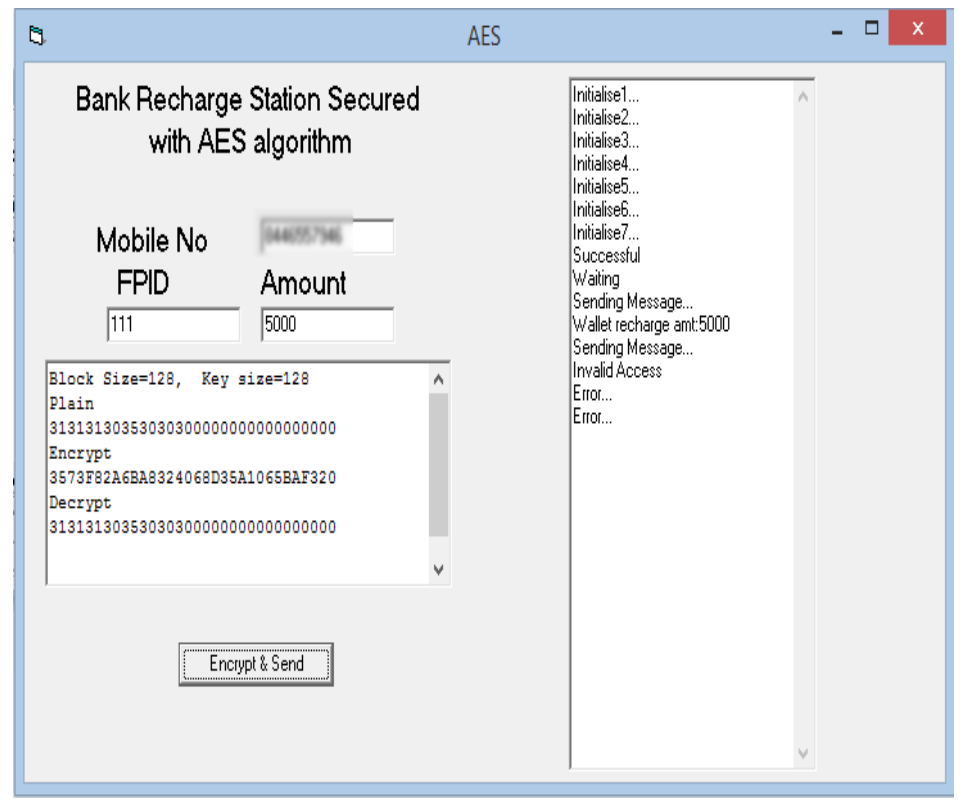

Fig.6. VB interface for the Bank Server

Now a day's different wireless payment method have been used. Mobile devices are generally integrated for the purpose of payment. The proposed digital wallet is for the purpose of payment which is highly secured using AES algorithm and fingerprint scanner. The fingerprint scanner makes it very easy for the user to make the payment. The required amount of bill is then automatically paid without any efforts. This method of payment makes the bill payment much easier.

\section{Conclusion}

Throughout the ages the method of payment have evolved and provided effective means for exchange of value. This has ultimately created the business world which has become integral part of everyone's life. The emergence of electronic world and the rise of eCommerce have forced to invent new payment methods. Without payment there is no business and eCommerce cannot exist without effective means of exchanging values.

The proposed work provides a very modern and advance way of payment technique through which the user can easily do billing process without much effort. The digital wallet thus provides a safe and secure way of payment which can be used in everyday life.

\section{References}

[1] R. Balan and N. Ramasubbu, "The Digital Wallet: Opportunities and Prototypes", Computer Network, Vol. 42, pp. 100-102, 2009.

[2] A. Illiev and S. Smith, "Private information storage with logarithmic-space secure hardware", in proceedings of IEEE international conference on Information Security, Management, Education and Privacy, pp. 156, 2004.

[3] Avinaash Kak and Keith Mayes, "AES: The Advanced Encryption Standard", in proceedings of IEEE international conference on Computer and Network Security, pp. 140-156, 2014. 
[4] M. Franklin and M. Yung, "Secure and efficient off-line digital money", in proceedings of IEEE international conference of International Colloquium on Automata, Language and Programming, pp. 256276, 1993.

[5] K.cMayes and K. Markantonakis, "Smart Cards, Tokens, Security and Applications", in proceedings of IEEE international conference on Information Security, Management, Education and Privacy, pp. 256, 2008.

[6] Benjamin M. Friedman, "Decoupling at the Margin: The Threat to Monetary Policy from the Electronic Revolution in Banking", in proceedings of IEEE international conference on Finance, pp. 14-26, 2000.

[7] M. Mary, N. Balakrishnan and S. Pratheeba, "A study on the Success Potential of Multiple Mobile Payment Technologies", in proceedings of IEEE international conference, 2010.

[8] M. Hashemi and E. Soroush, "A Secure m-Payment Protocol for Mobile Devices", in proceedings of IEEE international conference, May, 2006.

[9] Liu Shifeng and Chen Rong, "Wireless E-commerce Payment and its Development in China", IEEE Trans. Inf. Theory, vol. 58, no. 1, pp. 554-558, 2010.

[10] V.C.Sekhar and M.Sarvabhatla, "Secure Lightweight mobile payment protocol using symmetric key techniques," IEEE Transaction Communcation, vol. 54, pp. 469-478, 2012.

[11] Yan Li, Xiaoqiang Hu and Liang Zeng, "The application of mobile Agent in mobile payment," IEEE Transaction on Computer Science, vol. 3, pp. 1612 - 1616, 2011.

[12] Szu-Hui Wu and Chyan Yang, "Promoting Collaborative Mobile Payment by Using NFC-Micro SD Technology," IEEE Transaction on Computer Science, vol. 3, pp. 454 - 461, 2013.

\section{Authors' Profiles}

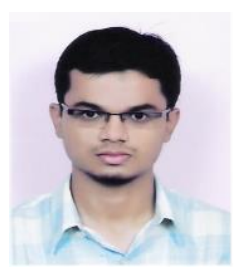

Harshal Rajesh Kanhekar (born April 10, 1991) is completing Master Degree in Engineering in Communication Network from D.Y.Patil Colllege of Engineering, Pune, India.

Mrs. Sayali N. Mane studied Electronics and Telecommunication Network. She has completed her Master Degree from Shivaji University. She is currently working as an Assistant Professor in Dr. D.Y.Patil College of Engineering, Pune, India.

How to cite this paper: Harshal R. Kanhekar, Sayali N. Mane,"Digital Wallet", IJWMT, vol.5, no.4, pp.62-68, 2015.DOI: 10.5815/ijwmt.2015.04.06 